\title{
INFLUENCIA DE LA INTELIGENCIA EMOCIONAL Y SOPORTE SOCIAL EN LA ORIENTACIÓN EMPRENDEDORA DE ESTUDIANTES INGRESANTES AL ÁREA DE CIENCIAS EMPRESARIALES DE UNIVERSIDADES NACIONALES DEL PERÚ Y ECUADOR
}

INFLUENCE OF EMOTIONAL INTELLIGENCE AND SOCIAL SUPPORT ENTREPRENEURIAL ORIENTATION OF INCOMING STUDENTS TO THE AREA OF BUSINESS ADMINISTRATION NATIONAL UNIVERSITY OF PERU AND ECUADOR

\author{
Ebor Fairlie F.* \\ efairlie@unmsm.edu.pe \\ Juan Carlos Lara $\mathrm{O}^{* *}$ \\ jclara@uleam.edu.ec \\ Guadalupe Toala $\mathrm{T}^{\text {***** }}$ \\ gtoala@uleam.edu.ec \\ Mario Moreira $\mathbf{M}^{* * * * * * *}$ \\ mmoreira@uleam.edu.ec \\ Evelyn Cano L********⿲二丨匕 \\ ecano@uleam.edu.ec

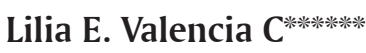 \\ lilyvalenciac@hotmail.com \\ Arturo Benavides R. \\ abenavidesr@hotmail.com
}




\title{
RESUMEN
}

Se ha realizado un estudio longitudinal que describe la influencia de la inteligencia emocional (IE) y soporte social (SS) en la orientación emprendedora (OE) de estudiantes que ingresan a la universidad, es decir, al iniciar su carrera profesional en la Facultad, luego de la formación académica; seguiremos la investigación cuando los estudiantes de las universidades nacionales del Perú y Ecuador de la carrera de Ciencias Administrativas finalicen su carrera en el 2017. Los objetivos se han cumplido como lo demuestra los resultados del estudio: A) conocer la orientación emprendedora (OE), inteligencia emocional (IE) y soporte social (SS) de los estudiantes al ingresar a la universidad; B) descubrir la evolución de OE, IE y SS al egresar de la carrera profesional; C) buscar la relación entre OE, IE y SS en los estudiantes ingresantes; y D) establecer comparaciones de la OE, IE y SS según variables demográficas, tales como sexo, edad y otros datos, en los que se han encontrado relaciones significativas entre estas variables y más que todo una semejanza en el comportamiento emprendedor de los estudiantes de ambos países. Se ha tomado la encuesta a 72 estudiantes peruanos de la UNMSM (la más representativa) y 233 del Ecuador que incluyen dos universidades solo de la Facultad de Ciencias Administrativas que son la Universidad Península de Santa Elena y la Universidad laica Eloy Alfaro de Manabí. A futuro, se propone ampliar la muestra con estudios transculturales y relacionarlos con las condiciones socio-económicas o en otros niveles educativos.

Palabras clave: Orientación emprendedora, inteligencia emocional, soporte social, emprendimiento.

\begin{abstract}
We carried out a longitudinal study describing the influence of EI and SS in the OE of students entering the university, that is, when you start your career in the Faculty, after the academic training, we will continue the investigation when students finish his career in 2017 between National Universities of Peru and Ecuador career management science. The objectives have been met as evidenced by the results of the study: A) Meet Entrepreneurial Orientation (EO), emotional intelligence (EI) and Social Support (SS) of students to enter the university; B) Discover the evolution of OE, IE and SS to graduate career; C) Find the relationship between OE and IE SS incoming students; and D) Establish comparisons OE, IE and SS according to demographic, such as sex, age and other data variables. Where they found significant relationships between these variables and most of all a similarity in entrepreneurial behavior of students in both countries. The survey has been taken 72 Peruvian students of Universidad Nacional Mayor de San Marcos (the most representative) and 233 of Ecuador including only 2 Universities Faculty of Administrative Sciences are the Universidad Península de Santa Elena and the Universidad Laica Eloy Alfaro de Manabí; in the future, it is proposed to extend the sample with cultural studies and relate to socioeconomic conditions or other educational levels.
\end{abstract}

Keywords: Entrepreneurial orientation, emotional intelligence, social support, entrepreneurship.

\section{INTRODUCCIÓN}

El estudio se ha desarrollado aplicando una encuesta validada a estudiantes ingresantes de la Universidad Nacional Mayor de San Marcos en Perú y la Universidad Península de Santa Elena en Ecuador.

Se han cumplido los objetivos de determinar la relación entre las variables de orientación emprendedora, inteligencia emocional y soporte social así como el de realizar las comparaciones entre ellas, encontrándose en ambos países una relación significativa como se describe en los resultados.
Los resultados demuestran que no hay diferencias entre ambos países, $(\mathrm{p}>0.05)$ y que los estudiantes ingresantes a la universidad, la mayoría no trabaja, no ocupa cargos directivos, aunque en un porcentaje mayor lo tienen los de Ecuador (80.6\% Perú y 88.6\% de Ecuador), porcentaje bajo en mejorar un negocio o empresa, y pocos tienen negocio propio o familiar.

Lo que llama la atención es la característica de alto emprendimiento que muestran tanto en Perú y Ecuador para desear "crear un negocio" en Perú 91.7\% y en Ecuador 89.8\% de los estudiantes universitarios. 
El análisis de regresión tiene como modelo la variable dependiente de OE y variable residual ponderada del país (Perú-Ecuador). El valor $\mathrm{F}$ total es 28354.9 para la intersección de OE, IE y SS, con un nivel de significación de 0.00 .

Así, se demuestra que IE y SS por separado influyen en la OE según el país de referencia

\section{MÉTODOS}

\section{Participantes}

La selección de los participantes fue de tipo intencional con criterio de inclusión tal como ser estudiante universitario ingresante el 2014 de la Facultad de Administración y haber completado adecuadamente los cuestionarios, de lo contrario quedaban eliminados.

La muestra quedó conformado por 72 estudiantes de la Universidad Nacional Mayor de San Marcos (UNMSM) y de Ecuador, 233, distribuido de la siguiente manera Universidad Estatal Península Santa Elena (UPSE), 129 y la Universidad Laica Eloy Alfaro de Manabí (ULEAM), 104.

La edad promedio de Perú es de 19 años (DS=1.8), con un mínimo de 16 y máximo de 29 , $100 \%$ solteros $43.1 \%$ mujeres y hombres $56.9 \%$; con estado de salud buena en $81.9 \%$.

En las universidades de Ecuador la edad promedio es 20 años (DS $=2.00$ ) comprendidas entre 17 a 30 años; mujeres, $61.9 \%$, y hombres, $38.1 \%$; solteros, $94 \%$, y estado de salud buena en $74.8 \%$.

\section{Procedimiento}

En ambos países se solicitó permiso al Decanato de la Facultad para realizar este proyecto de investigación. El criterio de inclusión para participar es haber ingresado el 2014, pertenecer al salón de clase del profesor investigador y responder a los cuestionarios, previo permiso solicitado.

Las instrucciones explicitan el anonimato y confidencialidad de los resultados, que en ningún caso serán individuales sino grupales y permitirán un diagnóstico para ayudarlos posteriormente. La duración de la administración fue de 15 minutos. Todos los cuestionarios fueron traducidos del inglés al español y luego se volvieron a traducir al inglés, siguiendo las técnicas de validación de traducciones, para asegurarnos el sentido exacto de los ítems.
Se realizó la revisión de los cuestionarios, descartando los incompletos, luego se codificaron las respuestas para crear la base de datos del SPSS U.21.

\section{INSTRUMENTOS DE MEDICIÓN}

\section{Datos demográficos y conducta emprendedora}

Solicitamos información demográfica sobre edad, género, estado civil y de salud con el objetivo de tener referencias de la muestra.

A continuación preguntamos sobre la conducta emprendedora, considerada esta como la actividad actual realizada en relación a la creación o mejoramiento de negocios o empresas, si trabaja o no actualmente, o de haber ocupado cargos directivos en un negocio o empresa, tener un negocio propio o familiar y sobre todo pensar en crear un negocio propio, preguntas categóricas que respondían con un sí o no.

\section{Orientación emprendedora (OE)}

Siguiendo a Kuratko (2007) sobre la orientación emprendedora en las organizaciones y cuyas dimensiones se refieren a riesgo asumido o toma de riesgo $(\mathrm{R})$, desde el punto de vista de la inversión y/o modificaciones riesgosas que realizan las empresas o los individuos.

Luego la innovación (I), como la nueva e imaginativa alternativa para idear o crear cambios. Por último tenemos la característica de la proactividad organizacional o de la empresa para actuar adecuadamente frente a la competencia.

Kuratko (2007) afirma que este planteamiento de orientación emprendedora (OE) que está dirigido a las organizaciones, también puede aplicarse a los individuos y obtener la OE de las personas, en este caso de los estudiantes ingresantes a la universidad, a través de las dimensiones:

- Toma de riesgo (R), toma de riesgo pasado $(\mathrm{RP})$ y toma de riesgo actual (RA).

- Innovación ( I ).

- Personalidad proactiva o proactividad (P).

Petrides y Furnham (2001) tienen un cuestionario compuesto de dos ítems para cada dimensión pero está dirigido a las organizaciones o empresas. Así que para cumplir nuestro objetivo, que es el de medir la orientación emprendedora en los individuos, en este caso, estudiantes universitarios, buscamos cuestionarios que hayan tenido 
validez y confiabilidad sobre toma de riesgos $(\mathrm{R})$, innovación (I) y proactividad (P), que describiremos a continuación.

\section{Toma de riesgo $(\mathrm{R})$}

La propensión al riesgo es medido en términos de frecuencia de conductas de riesgo, relacionados a la recreación, salud, finanzas, seguridad social. Esta escala desarrollada por Nicholson et al. (2005), explora la toma de riesgo en el pasado adulto y actual. El cuestionario consta de seis ítems para cada opción del pasado adulto y actual (RP y RA), calificada en una escala de Likert de 1 completamente en desacuerdo y 5 completamente de acuerdo. El puntaje máximo es de 30 para cada opción, luego se suma $\mathrm{RP}+\mathrm{RA} / 2$ y resulta un promedio de riesgo.

Esta escala mide la necesidad de logro, motivación de alcanzar el éxito, decisión, autonomía para tomar decisiones en las áreas de recreación, salud, finanzas, seguridad. Los investigadores encuentran relación de toma de riesgo (TR) con la edad, sexo y a través de los tipos de trabajo, también con el sector de negocios.

\section{Innovación (I)}

Para medir el trabajo creativo de innovación (I) utilizamos dos ítems de Dolan y Metcalfe (2012), relacionadas a la información del sujeto sobre la riqueza de ideas e imaginación. La calificación es de una escala de Likert que va de 1 poco frecuente y 7 frecuentemente. Así, el máximo puntaje es 14. El puntaje alto correspondería a sujetos originales, creativos de algo nuevo para tener éxito.

Los investigadores encuentran relación de (I) con bienestar subjetivo y podría mejorar la vida de las personas.

\section{Proactividad, personalidad proactiva $(\mathrm{P})$}

Para medir la proactividad (P) usamos "Proactive Personality Scale (PPS)" de Bateman T.S. y Grant J.M. (1993). Consta de 10 ítems calificados en una escala de Likert con puntajes que van de 1 , fuertemente de acuerdo, hasta 7 , fuertemente en desacuerdo, con un puntaje total de 70 .

Sujetos que tienen alto puntaje revelarían una disposición proactiva estable, que toma iniciativa personal en un amplio rango de situaciones y actividades, que desempeña una conducta proactiva al asumir la iniciativa para mejorar circunstancias habituales o creando nuevas situaciones (Seibert et al. 2001).

La personalidad proactiva ha sido relacionada con el desempeño y éxito en el trabajo.

Los ítems representan la percepción de autonomía, autoeficacia, flexibilidad en la toma de decisiones, responsabilidad por la propia conducta y reconocimiento de su producción.

\section{Inteligencia emocional (IE)}

Utilizamos la escala de Wong y Law (2002) (WLEIS), una versión china de auto reporte de la inteligencia emocional que consiste de 16 ítems. Contiene cuatro dimensiones que son equiparables o simétricas a las planteadas por Mayer y Salovey (1997) para IE:

- Autoevaluación emocional (SEA).

- Evaluación emocional de otros (OEA).

- Uso de las emociones (UOE).

- Regulación de las emociones (REA).

Cada dimensión consta de cuatro ítems y son calificadas en una escala de Likert de 1 , fuertemente en desacuerdo, a 7, fuertemente de acuerdo (Feng Kong et al. 2012).

Wong y Law (2002) realizan la validez y confiabilidad de la escala y concluyen que la prueba mide rasgos de personalidad relacionadas a la afectividad pero sin llegar a un cociente de IE (Bar On, 2001). Obtenemos un puntaje total de IE, sumando SEA+OEA+UOE+ROE cuyo máximo es 112 puntos.

\section{Escala de soporte social percibido (SS)}

El soporte Social (SS) fue evaluado con "MultiDimensional Scale of Perceived Social Support (MSPP) (Zinet, Dahlem, Zinet y Farley, 1988). Consta de 12 ítems relacionados a percibir el soporte social, distribuidos para evaluar tres fuentes de apoyo social percibido, de la familia $(F)$, de otras personas significativas $(O)$ y de amigos (A).

La calificación en una escala de Likert, oscila de 1 , completamente en desacuerdo, a 7, completamente de acuerdo; el puntaje total es de 84, y 28 para cada dimensión, luego se suman las dimensiones $(\mathrm{F}+\mathrm{O}+\mathrm{A}=\mathrm{SS})$. Altos puntajes del MSPSS indicarían percibir soporte social en algunas de las dimensiones y bajo puntaje está relacionado 
con depresión y ansiedad (Zimet, et al., 1988) y diferencias de género.

Osman et al. (2013) administran el MSPSS a 610 estudiantes universitarios entre 18 y 24 años, realizando análisis estadístico de confiabilidad e invarianza entre hombres, mujeres, correlación de factores y análisis de regresión así como la Teoría de Respuesta a Ítems (IRT). Así queda demostrada la validez de este instrumento que utilizamos en esta investigación.

\section{RESULTADOS}

Nos ocuparemos de los resultados de ambos países, Perú y Ecuador, de la conducta emprendedora, los datos descriptivos, confiabilidad y comparación de las variables de estudio: inteligencia emocional (IE), orientación emprendedora (OE) y soporte social (SS). Luego la correlación entre variables totales y dimensiones, así como el análisis factorial exploratorio (AFE) y el análisis de regresión.

\section{Conducta emprendedora de Perú y Ecuador}

Los resultados demuestran que no hay diferencias entre ambos países, $(p>0.05)$ y que los estudiantes universitarios ingresantes a la universidad, la mayoría no trabaja, no ocupa cargos directivos, aunque en un porcentaje mayor lo tienen los de Ecuador (80.6\% Perú y $88.6 \%$ de Ecuador), porcentaje bajo en mejorar un negocio o empresa, y pocos tienen negocio propio o familiar.

Lo que llama la atención es la característica de alto emprendimiento que muestran tanto en Perú y Ecuador para desear "crear un negocio" (en Perú $91.7 \%$ y en Ecuador $89.8 \%$ ) por parte de los estudiantes universitarios.

Consistencia del estudio, datos descriptivos y comparación entre Perú y Ecuador de las variables de estudio.

Los resultados se darán en base a las variables de estudio, orientación emprendedora (OE) y sus dimensiones: toma de riesgo pasado y actual $(\mathrm{R})$, innovación (I) y personalidad proactiva (P); inteligencia emocional (IE) y sus dimensiones: autoevaluación emocional (SEA), evaluación emocional otros (OEA), uso de las emociones (UOE) y regulación de las emociones (ROE); soporte social (SS) y sus dimensiones: otros significativos $(\mathrm{O})$, familia (F) y amigos (A).
En los estudios sobre autoinformes es necesario tener datos sobre su confiabilidad; en este trabajo usamos el alfa de Gronbach para realizar la confiabilidad que oscila de 0.79 a 0.71 , con un promedio total de 0.74, Según Aron et al. (2001: 567) se considera entre 0.70 a 0.90 para que la prueba sea útil (ver tabla 2). Esta investigación cumple con ese requisito, por lo tanto, los datos son confiables.

El tamaño del efecto es un parámetro de la población estimada en una muestra comparada con otra, diseñada para indicar la importancia práctica o clínica de un fenómeno de estudio. Los tamaños más comunes de efecto son el "d" de Cohen, que es la diferencia de medias estandarizadas para la comparación de dos grupos. En este estudio los valores "d" de Cohen oscilan de 0.0 a 0.4 que se consideran efectos pequeños (Meyer et al., 2003) y que interpretamos como que las medias estandarizadas de Perú y Ecuador se superponen en aproximadamente $100 \%$ si "d" es 0.0 y de $60 \%$ si "d" es 0.4 . Presentamos en la tabla 2 los estadísticos descriptivos, media y DS de las variables y dimensiones de estudio de Perú y Ecuador, así como también la comparación a través del " $t$ " de Student para muestras no aleatorias y de diferente tamaño y variables continuas.

Las diferencias significativas bilaterales $<0.05$ se ubican en las siguientes variables: toma de riesgo (R) a favor de estudiantes peruanos. En soporte social (SS), los estudiantes de Ecuador se sienten más apoyados en total y en especial por la familia (F) que los estudiantes peruanos. En cuanto a inteligencia emocional los estudiantes ecuatorianos tienen mayor puntaje en uso de las emociones y regulación de las emociones, mientras que los peruanos tienen más alta la evaluación emocional de otros. Además se presentan los baremos de la Escala Lickert en ambos países (anexo 1).

\section{Correlación y análisis de regresión de las variables de estudio}

La asociación entre variables continuas se realizan a través de la correlación de Spearman. En este estudio realizamos una matriz de correlación para ambos países entre las variables de estudio para determinar la asociación entre ellas (tabla 3) considerando un nivel de relación significativa a partir de $r=0.20$. Analizaremos cada variable y sus dimensiones: en la OE la dimensión de toma de riesgo $(R)$ es una variable aislada que no tiene relación con las otras, tal como lo demuestra el análisis factorial de componentes principales del estudio de Fairlie (2013). 
La innovación sí tiene relación con proactividad, autoevaluación emocional, uso de las emociones y regulación de las emociones; la personalidad proactiva con soporte social de otros y de la familia, así como la autoevaluación emocional, evaluación emocional de otros, uso de las emociones y regulación de las emociones; el soporte social de otros con el soporte familia y amigos, y las variables de inteligencia emocional están relacionadas entre sí, tal como lo demuestran los estudios de estudiantes universitarios chinos (Junqui Shi, 2007). Presentamos en el anexo 2 y 3 la matriz de correlación de Perú y Ecuador.

\section{El análisis factorial exploratorio}

El análisis de regresión tiene como modelo la variable dependiente de OE y variable residual ponderada del país (Perú - Ecuador). El valor $\mathrm{F}$ total es 28354.9 para la intersección de OE, IE y SS, con un nivel de significación de 0.00 .

Tabla 1. Criterios de información a b

\begin{tabular}{|c|c|}
\hline \multicolumn{2}{|l|}{ Criterios de información $a b$} \\
\hline *2 Log. de la verosimilitud restringida & 651.599 \\
\hline Criterio de información de Akaike (AIC) & 653.599 \\
\hline Criterio de Hurvich y Tsai (AICC) & 653.668 \\
\hline Criterio de Bozdogan (CAIC) & 656.693 \\
\hline Criterio Bayesiano de Schwarz (BIC) & 655.693 \\
\hline \multicolumn{2}{|c|}{$\begin{array}{l}\text { Los criterios de información se muestran en formatos de: } \\
\text { a. Variable dependiente total OE (Orientación Emprendedora) } \\
\text { b. Variable residual ponderada por país (Perú y Ecuador) }\end{array}$} \\
\hline
\end{tabular}

Realizamos el análisis factorial exploratorio por el método de la extracción de componentes principales a través de una matriz de componentes rotados de las variables IE, SS y OE y sus dimensiones de IE (SEA, OEA, UOE, REA), de SS (O, A, F) y de OE (I, P, R). El objetivo fue determinar si las dimensiones de cada variable que planteamos teóricamente en el estudio formaban los tres factores propuestos. Condujimos el análisis factorial de cada país por separado y juntos (Perú-Ecuador). Los resultados son de la tabla 3 , presentando la matriz de componentes rotados de ambos países que explican el $73.462 \%$ de la varianza total, que es significativa para los resultados porque dicha rotación ha convergido en cuatro componentes o factores extraídos.

El primer factor compuesto por la variable de inteligencia emocional (puntaje total) y sus cuatro dimensiones (SEA, OEA, UOE y REA); el segundo factor del puntaje total de soporte social (SS) y sus tres dimensiones (A, F, O), y el tercer factor compuesto por el puntaje total de orientación emprendedora (OE) y sus dimensiones de innovación (I) y personalidad proactiva (P) y; por último, el cuarto factor, aislado, la toma de riesgo. Los resultados del análisis factorial de cada país confirman que esta dimensión es un factor aislado de la orientación emprendedora en estudiantes universitarios de Perú y Ecuador pese a que $\mathrm{R}$ interviene en el puntaje total de OE.

Tabla 2. Correlaciones de Perú y Ecuador juntos

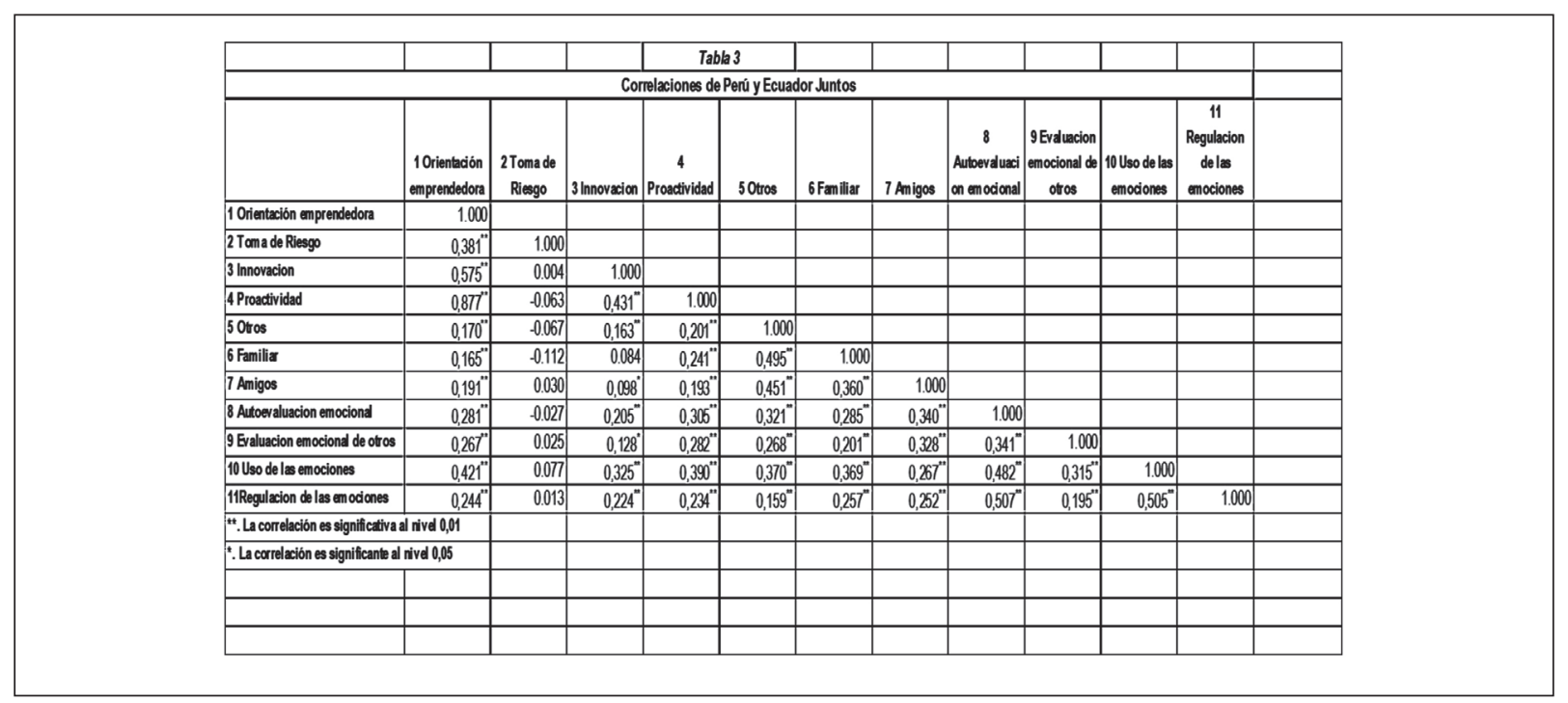


Tabla 3. Análisis factorial exploratorio para ambos países Perú-Ecuador

\begin{tabular}{|c|c|c|c|c|}
\hline \multicolumn{5}{|c|}{ Matriz de componentes rotadosa } \\
\hline & \multicolumn{4}{|c|}{ Componente } \\
\hline & 1 & 2 & 3 & 4 \\
\hline OE:Toma de Riesgo & .016 & -.067 & .049 & .963 \\
\hline OE: Innovación & .154 & .019 & .746 & -.085 \\
\hline OE: Proactividad & .194 & .157 & .871 & -.046 \\
\hline Total OE & .198 & .102 & .891 & .358 \\
\hline SS: Otros & .136 & .824 & .106 & -.052 \\
\hline SS: Familiar & .185 & .733 & .106 & -.173 \\
\hline SS: Amigos & .240 & .723 & .010 & .175 \\
\hline Total SS & .234 & .964 & .094 & -.018 \\
\hline IE: Autoevaluación emocional & .762 & .220 & .118 & -.052 \\
\hline IE: Evaluación emocional de otros & .495 & .276 & .114 & .167 \\
\hline IE: Uso de las emociones & .672 & .254 & .317 & .042 \\
\hline IE: Regulación de las emociones & .822 & .039 & .086 & -.056 \\
\hline Total IE & .938 & .258 & .207 & .036 \\
\hline \multicolumn{5}{|c|}{$\begin{array}{l}\text { Método de extracción: Análisis de componentes principales. } \\
\text { Método de rotación: Normalización Varimax con Kaiser. }\end{array}$} \\
\hline \multicolumn{5}{|c|}{ a. La rotación ha convergido en 4 componentes. } \\
\hline \multicolumn{5}{|c|}{ Varianza de cada factor. $39.296 / 15.213 / 10.638 / 8.315$} \\
\hline
\end{tabular}

\section{DISCUSIÓN}

Un objetivo del estudio fue encontrar las características de la conducta emprendedora a través de preguntas categóricas y comparar su asociación entre estudiantes universitarios ingresantes a la Facultad de Administración de la UNMSM y de dos universidades de Ecuador. El segundo objetivo fue realizar la confiabilidad de la muestra del estudio con el Alfa de Grombach y el efecto del tamaño con el "d" de Cohen. Un tercer objetivo fue comparar las variables de estudio, OE, IE y SS de los estudiantes peruanos y ecuatorianos, para determinar las semejanzas y diferencias de las variables investigadas. Un cuarto objetivo fue interrelacionar las variables de estudio, OE, IE, SS y sus dimensiones en una matriz de correlación para ambos países y por separado para cada país. Y por último, aplicar el análisis factorial exploratorio (AFE) y el de regresión múltiple para predecir si la OE está influenciada por IE y SS.

La confiabilidad de los instrumentos de medición está dentro de los límites aceptables, por lo que consideramos de utilidad este estudio. El tamaño del efecto "d" de Cohen es pequeño, dado que las medias de las variables se superponen e indican una muestra homogénea, para ambos países. El capital humano se ha convertido en un objetivo teórico a través del cual los investigadores pueden entender mejor el espíritu empresarial (entrepreneurship).

En este trabajo exploramos cómo los factores personales influyen en el comportamiento emprendedor individual, tomando en cuenta la "conducta emprendedora", considerada como la actividad realizada por los estudiantes universitarios ingresantes. Este emprendimiento podría contribuir al crecimiento económico a través de los esfuerzos para empezar o mejorar un negocio, que puede ser exitoso o no.

En este estudio encontramos que en la conducta emprendedora de Perú y Ecuador no hay diferencias significativas, entre estudiantes universitarios de ambos países son semejantes: ambos son de la Facultad de Administración, ingresantes, dependen de la familia, en un alto porcentaje no trabajan, no han realizado mejoras en algún negocio o empresa. Estas semejanzas nos llevan también al alto porcentaje del deseo de crear un negocio; como si la juventud universitaria de las Facultades de Administración estuvieran atentos para conseguir emprendimientos. Será necesario incentivar dicho deseo a través de la 
creación de un Centro de Emprendimiento, tal como lo propone Bouby Tolentino (2014), pero conceptualmente diferente. La creencia de que el espíritu empresarial es más que la mera creación de negocios, aunque esto es cierto, también lo es la característica de buscar oportunidades, así como tomar riesgos (riesgos pasados y presentes). El emprendimiento es un concepto que permite, de manera innovadora, integrar nuestra sociedad.

El grado de emprendimiento, considerado en el presente estudio como "orientación emprendedora" puede ser considerado como una aditiva función de tres dimensiones como el grado de innovación más el grado de toma de riesgo, más el grado de proactividad (Kuratko, 2007).

El grado de innovación es representado por el ítem de verse a sí mismo como original con habilidades para producir ideas nuevas y como alguien que tiene una imaginación productiva. No hay ahí diferencias significativas entre los estudiantes de Perú y Ecuador.

La toma de riesgo está en aspectos recreacionales, salud, profesión, financiero, de seguridad y social. Según los estudios realizados por Dubard Barbosa (citado por Kuratko 2007) indica que individuos con alto riesgo tienen altos niveles de intenciones de emprendimiento y eficacia en identificar oportunidades, mientras que individuos con bajo riesgo tienen altos niveles de eficacia en las relaciones y eficacia en la tolerancia. Los resultados del estudio indican que los estudiantes universitarios peruanos tienen más alto el puntaje de toma de riesgo.

La personalidad proactiva es determinada por encontrar mejorar, fuerzas para conseguir cambios, propuestas que se convierten en realidad, aspirar a ser ganador, identificar oportunidades, mejorar y hacer bien lo que se propone; creer en una idea y llevarla a cabo, características, que según el análisis factorial (Fairlie, 2013) pertenecen más al área afectiva. En el presente estudio no hay diferencias entre ambos países.

En la orientación emprendedora, que es la dimensión de la toma de riesgo, predomina en los estudiantes peruanos.

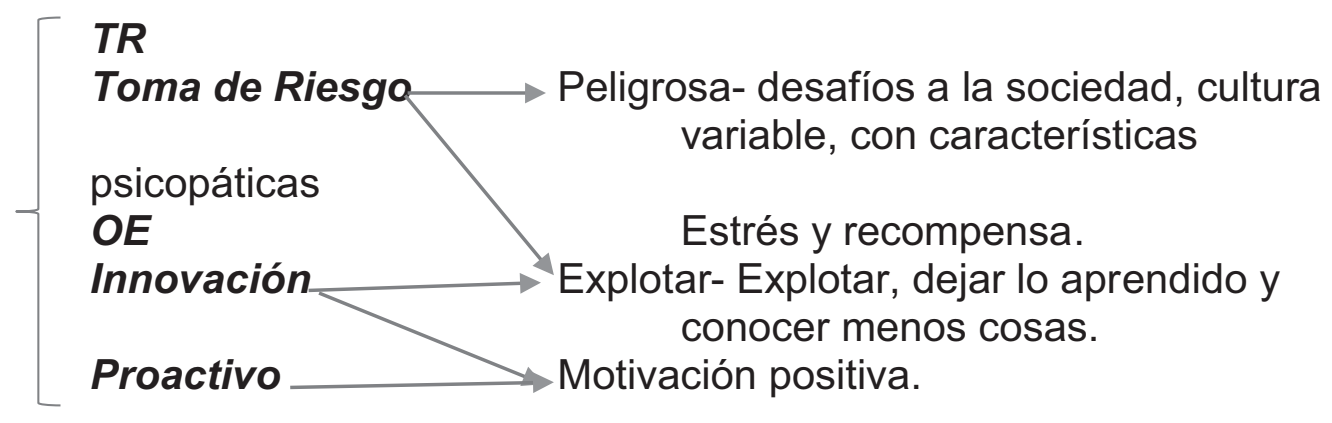

Fig. 1. Toma de riesgo

La toma de riesgo tiene una pequeña asociación con SS y con algunas dimensiones de IE como SEA (en autoevaluación emocional la correlación es negativa). No hay influencia de la IE y SS en la OE sobre todo porque hay contradicciones entre IE y toma de riesgo, mientras que I y proactividad presentan características positivas de la personalidad. La toma de riesgo se refiere a tomar decisiones peligrosas para sí mismo y con daño colateral para otros, porque desafía a la sociedad.

Según los estudios de Jessica Richard et al. (2015) sobre la toma de riesgo en adolescentes de 15 a 18 años, la TR aumenta el comportamiento ansioso y lleva a consecuencias potencialmente desastrosas, en las que el estrés y la recompensa interactúan y tiene mayor impacto en la toma de decisiones arriesgadas, sobre todo cuando hay ansiedad social.

Es así que el soporte social sirve de control sobre la toma de riesgo. Encontramos que los estudiantes ecuatorianos tienen menor puntaje de toma de riesgo y mayor apoyo social de la familia que produciría menos ansiedad social y menor estrés.

\section{Orientación emprendedora (OE)}

Kuratko (2007), cuando trata sobre la OE, manifiesta que diferentes combinaciones de 
dimensiones $(\mathrm{I}, \mathrm{P}, \mathrm{R})$ son posibles y un particular evento de emprendimiento, puede ser altamente innovativo con limitado riesgo y poca proactividad. Entonces hay un grado de emprendimiento (OE) referido a la extensión de innovación, riesgo y proactividad que pueden variar de acuerdo a las circunstancias, y no están íntimamente unidas. El grado de OE puede ser pensado como una función aditiva del grado de innovación + el grado de riesgo + el grado de proactividad. Esta conceptualización del grado del emprendimiento corresponde a cómo el individuo piensa actualmente sobre las situaciones de emprendimiento (Morris et al. 2008).

Kuratko al comentar sobre el líder emprendedor del siglo XXI, considera tres factores negativos que impregnan la personalidad del líder emprendedor dominan su conducta. El primer componente es la confrontación con la toma de riesgo, porque tiene que afrontar varios tipos de riesgo como el financiero, o estancarse en su profesión y con la familia y la sociedad. El segundo, es el estrés del emprendedor, que puede ser visto como una discrepancia entre las expectativas personales y la habilidad para encontrarlas, así como sus características de personalidad. Por último el engrandecimiento del yo, del líder emprendedor con un yo inflado, deseo por el éxito o un no realista optimismo. A todo estos factores los denomina la triada oscura del emprendimiento.

\section{Sobre OE}

Hansen et al. (2011), utiliza la escala de Covin y Slevin llamada análisis factorial confirmatorio (AFE) en sujetos de empresas pequeñas y medianas sobre $\mathrm{OE}$, analizando sus propiedades psicométricas a través de seis países, identificando el modelo óptimo para probar la invarianza de OE. El constructo del $\mathrm{OE}$ ha sido usado extensamente a través de la literatura, la definición e instrumento de medición ha generado debates sobre todo en lo que respecta a la dimensionalidad de las medidas. Muchos investigadores han eliminado ítems o en otros casos ha colapsado la escala en dos factores basados en procedimientos exploratorios, otros han focalizado una sola dimensión.

En este estudio sucede que la OE está compuesta de dos factores: uno que se refiere a la innovación y proactividad, y otro que es la toma de riesgo. Así sucede en la investigación anterior (Fairlie, 2013), donde la OE está compuesta por dos factores: 1) I+P y 2) R. Hansen encuentra que la toma de riesgo es el constructo que diferencia la $\mathrm{OE}$ en los seis países, con predominio de $\mathrm{R}$ en Estados Unidos sobre los otros seis países como Australia, Suecia, México, Indonesia, Holanda y Grecia.

Interpretando la toma de riesgo como el desear hacer mejoras en proyectos que tienen resultados inciertos o inusualmente con altas ganancias y pérdidas, Estados Unidos es el país con características más altas en toma de riesgo. Cuando comparamos Perú y Ecuador, esta variable de toma de riesgo es más diferenciada en el Perú, mientras que la innovación es la tendencia a experimentar cosas nuevas y ponerlas en práctica (Lumpkin y Dess 2001), la conducta proactiva refleja una propensión a actuar agresivamente hacia las empresas rivales con el propósito de favorecer oportunidades de negocio. Ambas variables I y $\mathrm{P}$ son factores que tienen ambos países (Perú y Ecuador), con correlaciones significativas.

\section{Toma de riesgo}

Según Nicholson et al. (2005) la propensión al riesgo se presenta en las áreas de recreación, salud, profesional, financiero, de seguridad y riesgo social, que construye la escala utilizada y describe sus propiedades psicométricas y su relación con variables demográficas, autorreporte biográfico, el NEO-PIR y el inventario de personalidad de los cinco factores. La propensión al riesgo fue relacionada con la edad, el sexo y la profesión. En este estudio encontramos dos aspectos relevantes: en primer lugar, hay diferencias significativas en Perú y Ecuador, siendo la OE a favor del Perú; y en segundo lugar, en el análisis factorial exploratorio de los componentes principales se presenta un factor aislado de la OE.

Las investigaciones realizadas han estudiado la personalidad y capacidades que subyacen en el emprendimiento, pero han ignorado los aspectos negativos de la personalidad emprendedora tales como la agresividad, el narcisismo, la crueldad e irresponsabilidad que se presentan en esta variable de toma de riesgo . Dadas las repercusiones costosas de esta característica instamos a realizar más estudios sobre este tema, sobre la naturaleza y causa de la iniciativa empresarial (Miller, 2014).

Kim-Yin Chang et al. (2015), en un estudio sobre la carrera profesional, liderazgo y emprendimiento de estudiantes universitarios de pregra- 
do, encuentran que los rasgos de personalidad de motivación por el trabajo, la baja aversión al riesgo y la personalidad proactiva tienen relación con el emprendimiento y liderazgo. Notar que se refieren en aversión al riesgo, factor aislado, que según nuestro estudio no está considerado dentro de la OE.

Según Richard (2015) la toma de riesgo aumenta el comportamiento ansioso en adolescentes y lleva a consecuencias potencialmente desastrosas, de acuerdo al estudio experimental realizado en adolescentes de 15 a 18 años. Concluye con tres aspectos esenciales: primero, la magnitud de recompensa tiene un mayor impacto en la toma de decisiones arriesgadas; segundo, el efecto del tiempo de reacción es más bajo en aquellos sujetos con alta ansiedad social; y por último, los adolescentes con alta ansiedad social evidencian mayor tiempo de reacción en juicios más negativos. En conclusión la toma de riesgo de pronta recompensa elevan el rasgo de ansiedad social.

\section{Innovación}

Según Dolan y Metcalfe (2012) las variables de innovación (I) son la originalidad, el sujeto se percibe así mismo como alguien que es original, con nuevas ideas; y la imaginación, se percibe como alguien con gran actividad imaginativa.

La OE como capital humano es vista como conducta emprendedora y según Kuratko (2007) "El emprendimiento es una función del emprendedor $\mathrm{E}=\mathrm{f}(\mathrm{e})$. El emprendimiento es un proceso dinámico de visión, cambio y creación, ello requiere una aplicación de energía y pasión hacía la creación e implementación de nuevas ideas y soluciones creativas. La innovación es un ingrediente esencial en el emprendimiento personal que contribuye al desarrollo económico nacional".

Según Bledow et al. (2009) décadas de investigación de diferentes disciplinas y niveles de organización han producido una gran cantidad de conocimiento sobre cómo surge la innovación y los factores que la inhiben o facilitan. Proponen una integración dialéctica de este conocimiento para obtener una explicación más válida de la innovación y plantea la ambidestreza organizacional, que se refiere a la capacidad de una organización para aprovechar las oportunidades existentes en el mercado de manera eficiente y, a la vez, proponer soluciones creativas e innovadoras para satisfacer las demanda del mercado.
En nuestro estudio los niveles de innovación son altos en ambos países.

\section{Proactividad}

Según Seibert et al. (2001) la personalidad proactiva está relacionada al éxito en la carrera profesional y en el trabajo. Fuller et al. (2009) añade que la personalidad proactiva es una disposición estable que toma la iniciativa personal en un amplio rango de situaciones y actividades, para mejorar circunstancias corrientes o creando nuevas situaciones. La personalidad proactiva no solamente toma la iniciativa para hacer avances en su lugar de trabajo, también lo hace en su propia carrera, así como desarrollan actividades consultando con otros, estableciendo redes de comunicación. Está relacionada con el éxito cuando se es líder, con el rendimiento en el trabajo, es decir con la conducta proactiva, y la motivación de logro y adaptabilidad al medio que lo rodea. Esta concepción de personalidad proactiva es tomada por Bateman y Grant (1993) para realizar la encuesta que utilizamos en este trabajo y cuyos resultados no difieren en ambos países.

Tabla 4. Diferencias de variables y dimensiones de OE, IE y SS entre estudiantes peruanos y ecuatorianos

\begin{tabular}{|c|c|c|}
\hline Variable & Perú & Ecuador \\
\hline OE & $\begin{array}{c}\text { Toma de riesgo } \\
(\mathrm{R})\end{array}$ & \\
\hline IE & $\begin{array}{c}\text { Evaluación emocio- } \\
\text { nal de otros (OEA) }\end{array}$ & $\begin{array}{c}\text { Uso de las emocio- } \\
\text { nes (UOE) }\end{array}$ \\
\cline { 2 - 3 } & $\begin{array}{c}\text { Regulación de } \\
\text { emociones (ROE) }\end{array}$ \\
\hline SS & Familia (F) \\
\hline \multicolumn{2}{|c|}{ Orientación Emprendedora (OE), Inteligencia Emocional (IE) y } \\
\multicolumn{2}{|c|}{ Soporte Social (SS) } \\
\hline
\end{tabular}

En la tabla 4 se observa que en cuanto a la inteligencia emocional hay diferencias entre estudiantes de ambos países, la interpretación sobre la evaluación emocional de otros (OEA) que predomina en estudiantes peruanos, se ha encontrado en diferencias de sexo siendo menor en hombres, así como también que tienen mayor conciencia de sí mismos y de los otros (Junqi Shi, Lei Wang, 2007). Los estudiantes ecuatorianos manejan en mayor medida el uso de las emociones (UOE) que según Junqi es mayor en hombres, relacionado con la percepción de síntomas positivos y mayor concientividad. En cuanto a la regulación emo- 
cional (ROE) está asociada con el mejor manejo de afrontamiento al estrés. En cuanto al soporte social (SS) los estudiantes de Ecuador perciben el apoyo familiar (F) con mayores puntajes.

Estas diferencias podemos sintetizarlas de la siguiente manera: el estudiante peruano tiene más alto puntaje de toma de riesgo (R) y propensión a realizar actos atrevidos con una percepción emocional de los otros que lo diferencia de sí mismo. El estudiante ecuatoriano percibe mayor dependencia de la familia y usa sus emociones, regulando adecuadamente el estrés situacional, siendo más tolerante en sus relaciones interpersonales.

En cuanto a las relaciones entre las variables encontramos que la toma de riesgo es una variable aislada que no correlaciona con las otras de $\mathrm{OE}$, como es la innovación y la personalidad proactiva, lo que pone en duda lo planteado por Kuratko (2007). Mientras que las variables de inteligencia emocional correlacionan entre como la innovación y personalidad proactiva. La variable de soporte social y sus dimensiones correlacionan entre sí.

El análisis de regresión múltiple nos indica que la IE y SS influyen en la OE, siempre y cuando sea referido a ambos países.

\section{CONCLUSIONES}

1. Analizando las características demográficas de los estudiantes universitarios de la Facultad de Administración de Perú y Ecuador encontramos que en el país vecino hay más mujeres y la edad es ligeramente mayor, y que en Ecuador el sistema de ingreso a la universidad cumple dos condiciones, tienen que ser bachilleres y aprobar el curso pre-universitario.

2. No hay diferencias significativas en la conducta emprendedora entre estudiantes peruanos y ecuatorianos. Pero lo importante es que el porcentaje de intención de crear un negocio o empresa es muy alto en ambos países. Como si la juventud universitaria de la Facultad de Administración estuviera deseosa, atenta a conseguir emprendimientos. Se podría satisfacer ese anhelo a través de la creación de un centro de emprendimiento.

3. El presente estudio demuestra apoyo para la confiabilidad de los datos obtenidos y validez de los instrumentos utilizados, como el cuestionario de inteligencia emocional (IE) y sus cuatro dimensiones, el autoinforme de soporte social (SS) y sus tres dimensiones, y el de orientación emprendedora (OE) con dos dimensiones, una Innovación (I) y Proactividad (P) que tienen una estructura estable y otra dimensión la toma de riesgo que se presenta separado de la orientación emprendedora.

4. La comparación entre Perú y Ecuador la vamos a ver a través del efecto del tamaño "d" de Cohen que es pequeño y que nos indicaría el predominio de la semejanza de promedios estandarizados en ambos países. Las diferencias estarían en la dimensión de toma de riesgo y autoevaluación emocional en peruanos, y a favor del país vecino, las dimensiones de apoyo familiar, uso de las emociones y regulación emocional.

5. La relación entre variables es significativa y demuestra que Inteligencia emocional y sus dimensiones se relacionan, así como soporte social. En cuanto a orientación emprendedora, la variable que no tiene relación significativa o es negativa es la toma de riesgo. El análisis factorial exploratorio confirma estas relaciones al encontrar cuatro factores: primer factor es inteligencia emocional (IE) con sus dimensiones; el segundo es el soporte social (SS) y sus dimensiones, el tercero, la orientación emprendedora y las dimensiones de innovación y proactividad; y el cuarto factor es la toma de riesgo.

6. El análisis de regresión múltiple revela que la inteligencia emocional y soporte social influye en la orientación emprendedora, no así en la toma de riesgo cuando se analiza cada universidad de Perú y en las de Ecuador.

\section{LIMITACIONES}

- Hay sin embargo algunas limitaciones en nuestro estudio. Primero, estos cuestionarios de IE, SS y OE son autoinformes y no puede evitarse el sesgo de deseabilidad social.

- Casi son preguntas directas acerca de la conducta emocional y apoyo social y emprendimiento.

- El sujeto podría ser entrenado para responder adecuadamente si quiere quedar bien con los otros.

- Es la primera vez que se usa en nuestro medio cultural Perú y en el Ecuador. 
- Si bien los resultados son confiables, las relaciones entre las variables aceptables y el análisis factorial explica el $73 \%$ de la varianza, estos resultados no pueden generalizarse porque la muestra no es aleatoria, es solo de estudiantes universitarios de la carrera de administración $\mathrm{y}$ afines.

- Los investigadores en el siglo XXI consideran la orientación emprendedora con las dimensiones de innovación + proactividad + toma de riesgo. De acuerdo a nuestro estudio planteamos que la OE debe ser un factor y otro la toma de riesgo.

\section{REFERENCIAS BIBLIOGRÁFICAS}

Aron, Arthur and Elaine Aron (2001). Estadística para Psicología. Pearson Education S.A., Buenos Aires.

Bateman, T.S. and Crant, J.M. (1993). The proactive componet of organizational behavior. Personal Psychology. 14, 103 - 118.

Bledow, Ronald; Frese, Michael; Anderson, Niel; Erez, Miriam, y Farr, James. 2009. A dialectic perspective on Innovation. En: Múltiplle pathways and ambidexterity. Vol.2 (3), pp. 305-337.

Bouby Tolentino, Francisca. (2014). Fomento del Emprendimiento Empresarial y Formación Profesional Universitaria: Centro de Emprendimiento en la FCA, UNMSM. En: Gestión en el Tercer Milenio. Vol. 17 - 1, N³3.

Dolan, P. y Metcalfe, R. 2012. The relationship between innovation and subjective weebeing. En: Research Policy. 41, pp. 1489-1498.

Fairlie E. (2013). El valor de la marca como estrategia para incrementar la capacidad emprendedora en estudiantes de universidades nacionales del área de Ciencias Empresariales. Tesis.

Kong, F., Zhao, J., You, X. (2012). Emotional intelligence and life satisfaction in chinese university students: The medianting role of self - esteem and social support. En: Personality and Individual Differences. 53, 1039 - 1043.

Fuller B. Jr., y Marler, L.E. (2009). Chang driven by nature: A metaanalytuc review of the proactive personality literatura. En: Journal of Vocational Behavior. 75, pp. 329-345.
Hansen. J. D.; Deitz, G.; Tokman, M.; Marino, L.; y Weaber, M. (2011). Cross - national invariance of the entrepreneurial orientation scale. En: Journal of Business Venturing. 26, pp. 61-78.

Shi, J., Wang, L. (2007). Validation of emotional intelligence scale in chinese university students. En: Personality and Individual Differences. 43, 377 - 387.

Kim-Yin Chan; LLy, Marilyn A.; Chemyshenko, Oleksandr; Mon Ho, Yoko Loo. (2015). Personality and entrepreneurial, professional and leadership motivations. En: Personality and Individual Differences. Vol. 77, pp. 161-166.

Kuratko, D. F. (2007). Entrepreneurial Leadership in the 21 st Century: Guest Editor's Perspective. En: Journal of Leadership \& Organizational Studies. 13 (4), pp. 1-11.

Lumpkin, G. \& Dess, G. (2001). Linking two dimensions of entrepreneurial orientation to firm performance: the moderating role of environment and industry life cycle. Elsevier. New York.

Mayer, J. \& Salovey, P. (1997). What is emotional intelligence?. Basic Books. New York

Meyer, G.; Mc-Grath, R.; y Rosenthal, R. (2003). Basic Effect Size Guide with SPSS and SAS Syntax. Last updated january 13.

Miller, Danny. (2014). A downside to the entrepreneurial personality? En: Entrepreneurship Theory and Practice. Vol. 39 (1), pp. 1-8.

Morris, M.; Kuratko, D. \& Covin, J. (2008). Corporate entrepreneurship \& innovation. Cengage learning. USA

Nicholson, N.; Seoane, E.; Fenton; O' Creevy; y Willman, P. 2005. Personality and domain specific risk taking. En: Journal of Risk Research. 8 (2), pp: 157-175.

Osman A, Downs WR, Barrios FX, Kopper BA, Gutierrez PM, Chiros CE. Factor structure and psychometric characteristics of the Beck Depression Inventory-II. J Psychopathol Behav Assess. 1997;19:359-76.

Petrides, K. V., y Furnham, A. (2001). Trait Emotional Intelligence: Psychometrc Investigation 
With Reference to Established Trait Taxonomies. En: European Journal of Personality. 15; pp. 425-448.

Richard, Jessica; Patel, Nilan; Zegarelli, Tereza; Mc Pherson, Laura; Lequez, C.W.; Ernst, Mónique. (2015). Social Anxiety, acute social stress and reward paramenters interact to predict risky decisión-marking among adolescents. En:Journal of Anxiety Disorders. 29 de enero del 2015, pp. 25-34.

Seibert, S.E.; Kraimer, M.L.; y Grant, J.M. (2001). ¿What do practive people do?. A longitudi- nal model linking proactive personality and carcer success. En: Personality Psychology. 54, pp. 845-874.

Wong, C. S., y Law, K. S. (2002). The effects of leader and fallower emotional intelligence on performance and attitude: An exploratorio study. En: The Leadership Quarterly. 13, pp. 243-274.

Zinet, G. D.; Dahlem, W.; Zinet, S.G.; y Farley, G. K. (1988). The Multidimensional Scale of Perceived Social Support. En: Journal of Personaliity Assessment. 52, (1), pp. 30-41. 